\title{
Design of Reconfigurable Uniform Digital Filter Banks based on Coefficient Decimation Method_Review
}

\author{
Ibtihaj H. Qadoori \\ University of Baghdad, \\ College of Engineering, \\ Department of Electrical Engineering
}

\author{
Mahmood A. K. Abdulsattar \\ University of Baghdad, \\ College of Engineering, \\ Department of Electrical Engineering
}

\begin{abstract}
With the technologies of wireless communication like cognitive radio (CR), analysis filter banks (FBs) are used to adopt two crucial tasks; channelization followed by spectrum sensing. The channelizer is used to obtain separate channels from the wideband digital input signal at different intervals of time. The three main requirements in the channelizer are reconfigurability, low complexity and flexibility. The coefficient decimation technique for reconfigurable FIR filters was recently discussed as a filter structure with low computational complexity. In this brief, the most important types of FBs based on Coefficient Decimation Method (CDM) that used in a uniform channelization with comparison among them based on their multiplication complexities and their flexibilities, are discussed.
\end{abstract}

\section{General Terms}

Digital Signal Processing, Finite Impulse Response Filters.

\section{Keywords}

Filter banks, Coefficient decimation method, Low Complexity, Flexibility, reconfigurability.

\section{INTRODUCTION}

Finite impulse response (FIR) filters and Filter Banks (FBs) are commonly performed in digital signal processing and are favored over their infinite impulse response equivalents because of their intrinsic phase linearity and stability features. Filter banks are basically divided into analysis banks and synthesis banks [1]. The analysis part of $M$-channel FB consists of analysis filters that separate the input signal into $M$ subband signals. Likewise, the synthesis part of $M$-channel FB contains $M$ synthesis filters that combine $M$ signals forming a reconstructed signal. Analysis FBs are used in the base station receivers of wireless communication for channelization intentions to extract the frequency channels from wideband input signal [2]. Analysis FBs are performed in cognitive radios to adapt channelization, and spectrum sensing, to detect the radio channels existence or absence [3], [4]. In the applications of resource constrained like battery-powered handsets of mobile Cognitive radio (CR), implementation of low complexity FB is preferred to certify efficient utilization of an inadequate available resources [2]. Many methods were proposed in literature to design and implement reconfigurable filter banks. In [1] the Discrete Fourier Transform Filter Bank (DFTFB) is performed as analysis uniform channelization FB. It contains prototype LowPass (LP) filter in polyphase form. The desired bands are obtained using the operation of Inverse DFT (IDFT). DFTFB is not capable of extracting different bandwidths channels (nonuniform channels) since they considered modulated FBs of equal bandwidth similar to that of the prototype LP filter. In [5], a
Coefficient Decimation Method (CDM) was proposed to achieve reconfigurable low complexity FIR filters. This method is capable of generating variable frequency responses using fixed coefficients of filter by applying two operations of coefficient decimation, the first used to vary the prototype filter passband width (abbreviated as CDM-II), the second used to generate multi-channel frequency responses (abbreviated as CDM-I). In [6], a Filter Bank based MultiStage Coefficient Decimation (MS-CDFB) was proposed that can offer a changeable sensing resolution in cognitive radios spectrum sensing. The MS-CDFB multiplication complexity is one-seventh that of DFTFB. The estimated power spectrum accuracy of the MS-CDFB sensor is better than DFTFB sensor. In [7], a new DFTFB was offered, that can be used to perform channels of multi-channel bandwidth. This architecture is reconfigurable and Coefficient Decimation (CD) based. The resulting filter passband width equals to the original filter passband width multiplied by $M$. In [8], a Modified Coefficient Decimation Method (MCDM) was proposed to achieve reconfigurable filters with improved flexibility of frequency response and twice the resolution of center frequency as compared with conventional CDM. The higher reconfigurability degree can be provided by this method as compared to the CDM. The filters resulted by this method show higher attenuation in the stopband while comparing with those achieved by conventional CDM, with lower complexity because of lower order of modal filter requirement in the MCDM. In [9], a new method for designing channel filter based on the combination of the MCDM-II and CDM-II was proposed and named as Improved Coefficient Decimation Method II ( ICDM-II ). Lower complexity can be realized by this channel filter when compared with other CDM channel filters like [10]. Once both are considered same specifications, this channel filter appears superior TBW and characteristics of stopband when compared with CDM channel filters. In [2], a uniform FB that combines MCDM-I with the conventional CDM-I to realize desired subbands, this method is named Improved Coefficient Decimation Method I and abbreviated as ICDM-I. In this design technique, wide-TBW masking filters [11] are employed to extract desired subbands individually from the frequency responses of multi-band achieved by performing ICDM-I operations on the modal filter. In this paper a revision to coefficient decimation based filter banks is presented and a unified design example of uniform subbands is performed to examine the complexity and flexibility of these types of FBs.

In Section 2, a revision of the coefficient decimation methods and a description of every approach is presented. A uniform channelization design example is presented in section 3 with a complexity and flexibility comparison. The conclusion is presented in section 5 . 


\section{REVISION TO COEFFICIENT DECIMATION METHODS}

The coefficient decimation method is used to obtain sub channels from the wideband digital input signal with reduced complexity by a factor of $(1 / M)$, where $M$ is the decimation factor.

\subsection{Coefficient Decimation Method I (CDM-I)}

If each $M$ th coefficient of an FIR filter is unaltered and the others are set to zeros [5], a frequency response like upsampling images is obtained and this process is named a coefficient decimation method I by a decimation factor of $M$ and abbreviated by CDM-I. The resulting filter frequency response expression is

$$
H^{\prime}\left(e^{j w}\right)=\frac{1}{M} \sum_{k=0}^{M-1} H\left(e^{j\left(\omega-\frac{2 \pi k}{M}\right)}\right)
$$

From (1), it can be observed that, the resulting $M$ scaled frequency spectrum copies are presented at multiples of $(2 \pi / M)$. After scaling the filter output by $M$, the original signal can be recovered.

\subsection{Coefficient Decimation Method II (CDM-II)}

After applying CDM-I by $M$ factor, the resultant filter reserved coefficients can be gathered by removing in between zeros, the resultant lowpass response that has TBW $M$ times the modal filter's TBW can be found. This process is abbreviated as CDM-II [12]. After performing the CDM-II, a lowpass response with passband width $M$ times the base passband width is achieved.

\subsection{Modified Coefficient Decimation Method I (MCDM-I)}

Now if each $M$ th coefficient of FIR filter is unaltered and the sign of each alternate unaltered coefficient is inverted. The rest coefficients are substituted by zeros [8]. The resultant FIR filter frequency response is

$$
H^{\prime}\left(e^{j \omega}\right)=\frac{1}{M} \sum_{k=0}^{M-1} H\left(e^{j\left(\omega-\frac{\pi(2 k+1)}{M}\right)}\right)
$$

The resultant multibands center frequencies are at $(2 k+1) \pi / M$, where $k$ is an integer number ranging from 0 to $M-1$. This method is named modified coefficient decimation method I (MCDM-I). It can be seen in (2), that the center frequency resolution of the resulting multibands is $\pi / M$.

\subsection{Modified Coefficient Decimation Method II (MCDM-II)}

After applying MCDM-I by $M$ factor, if the resulted filter reserved coefficients are gathered by removing in between zeros, then a highpass response is achieved with passband and TBWs equal to that of modal filter multiplied by $M$. This process is abbreviated as MCDM-II [9].

\subsection{Improved Coefficient Decimation Method II (CDM-II)}

The improved coefficient decimation method II (ICDM-II) combines CDM-II and MCDM-II processes [9]. By applying ICDM-II operations, different lowpass and highpass responses can be achieved with various passband widths. Uniform frequency bands and nonuniform bands are achieved from the highpass and lowpass responses using addition or subtraction of the spectrum, but no masking filters are used.

\subsection{Improved Coefficient Decimation Method I (CDM-I)}

A uniform FB can be provided from MCDM-I and CDM-I combination to obtain uniform subbands (and abbreviated as ICDM-I). In this design technique, low order FRM filters [11] are needed to extract the desired subbands from the multibands obtained from performing ICDM-I operations on the modal filter.

\subsection{Improved Coefficient Decimation Method (ICDM)}

In the uniform channelization, the ICDM [13], employs the combination of ICDM-I, spectral subtraction, operation of complementary response and FRM operations for extracting the specific FB subbands. In the nonuniform channelization, the ICDM employs the combination of ICDM-II and ICDM-I to extract the specific subbands.

In all coefficient decimation operations based $\mathrm{FB}$, the increasing values of $M$ reduce FB complexity. An $N$-order undecimated FIR filter has $(N+1)$ multiplications, while the number of multiplications $\left(N_{m}\right)$ for decimated FIR filter will be

$$
N_{m}=\left\lceil\frac{N+1}{M}\right\rceil
$$

The order of FIR filter used in the design of all types of CDM based FBs can be calculated using [14]

$$
N=-\frac{4 \log _{10}(10 * \delta p * \delta s)}{3\left(f_{s}-f_{p}\right)}-1
$$

Where $f_{p}$ is the desired passband frequency and $f_{s}$ is the desired stopband frequency (normalized in the range $0-1$, with 1 corresponding to the Nyquist frequency) and $\delta p$ is the desired passband peak ripple and $\delta s$ is the desired stopband peak ripple.

It will be observed in the design example in section 3 that, the stopband attenuation deteriorates after coefficient decimation and this deterioration should be considered in the filter order calculation. The mathematical expression for SA deterioration is

$$
\delta_{s(\text { modal })}=\frac{\delta_{s(\text { final })}}{M}
$$

where $\delta_{s \text { (modal) }}$ is the modal filter's stopband attenuation and $\delta_{s(\text { final })}$ is the resulted filter stopband attenuation. So it can be derived from (4) and (5) that in order to keep the SA within the desired value of $\delta_{s}$, the overdesigned modal filter minimum order should be given by [9]

$N=\left[\frac{-4 \log _{10}\left(10 \delta_{p} \delta_{s}\right)}{3\left(f_{s}-f_{p}\right)}-1\right]+\frac{4 \log _{10} M}{3\left(f_{s}-f_{p}\right)}$

The properties of each method can be further explained using an illustrative design example in section 3 . 


\section{UNIFORM CHANNELIZATION DESIGN EXAMPLE}

The design example in this section consists of eight fixed channel stacking, as shown in Fig 1, in which only five real channels within the Nyquist frequency are considered and the rest channels are in the imaginary part.

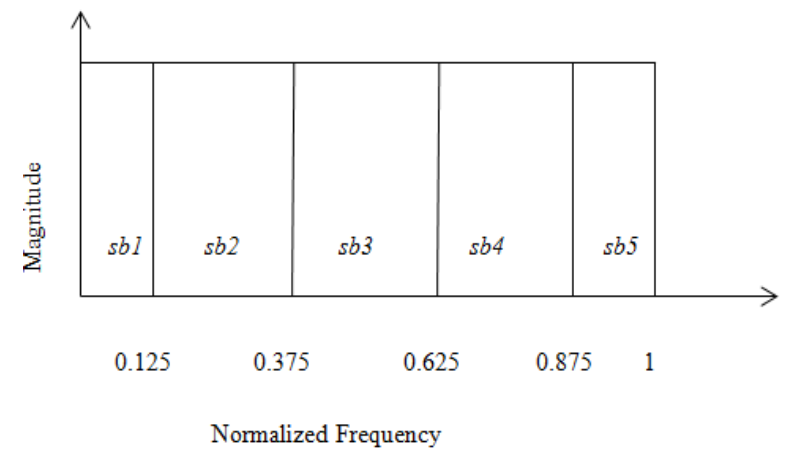

Fig 1: Uniform Channelization of Eight Channel Design Example

Four types of FBs will be used to extract the five subbands in Fig 1 based on the coefficient decimation method. The CDM can be used as a low complexity alternative to a uniform DFTFB. The modal filter is designed using the frequency edges of $\left(f_{p}=0.1125, f_{s}=0.1375\right)$, and the passband and stopband specifications $(\delta p=0.1 \mathrm{~dB}$ and $\delta s=-40 \mathrm{~dB})$. The modal filter's length is calculated using (3) as 161 and its frequency response is shown in Fig 2 (a). For obtaining sb1, the modal filter itself can be used. In order to obtain sb5, perform CDM-I on the modal filter using, $M=2$ as shown in Fig 2 (b) and subtract the frequency response of the modal filter from it. Sb3 can be obtained after performing spectral subtraction to the frequency response obtained after performing CDM-I, using $M=2$ and the frequency response obtained after performing CDM-I, using $M=4$ (Fig 2 (c)). $\mathrm{Sb} 2$ and $\mathrm{sb} 4$ can be obtained from the complementary response of Fig 2 (c) which can be separated by applying isolated masking filters of low order (18) as shown in Fig 2 (d).

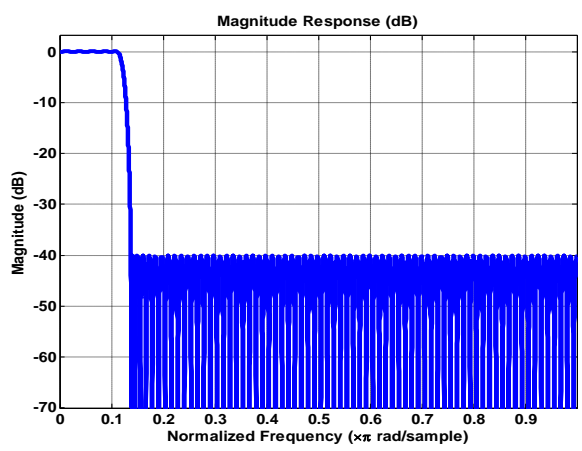

(a)Modal Filter

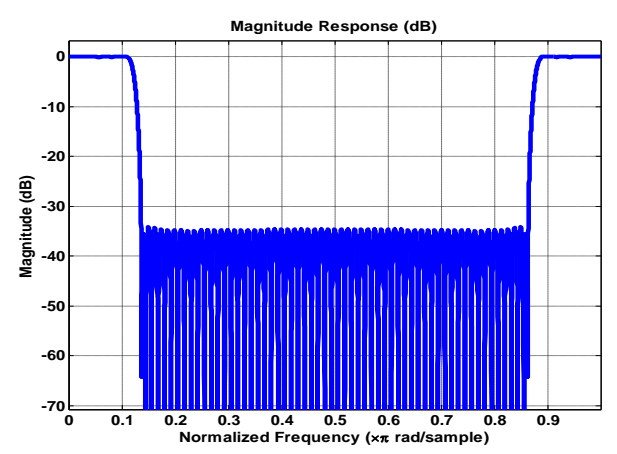

(b) CDM-I, $M=2$

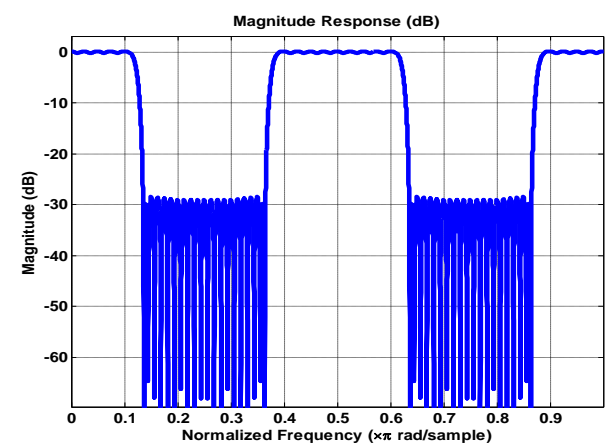

(c) CDM-I, $\boldsymbol{M}=\mathbf{4}$

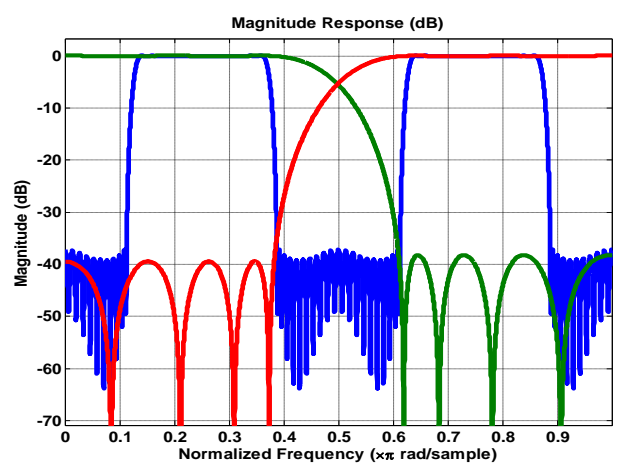

(d) Complementary Response of (c)

Fig 2: CDM Operations

The MCDM-I based FB can be employed for uniform channelization of Fig 1. The modal filter can be designed using the same specifications used in the CDFB. The modal filter itself can be used to get sblas shown in Fig 2 (a). After performing MCDM-II, using $M=1$ on the modal filter, a frequency response of Fig 3 (a) is obtained, which represents the frequency response of $\mathrm{sb} 5 . \mathrm{Sb} 2$ and $\mathrm{sb} 4$ can be obtained after performing MCDM-I on the modal filter, using $M=4$ as shown in Fig 3 (b). Whereas, sb3 is obtained after performing MCDM-I on the modal filter, using $M=2$ as shown in Fig 3 (c). 


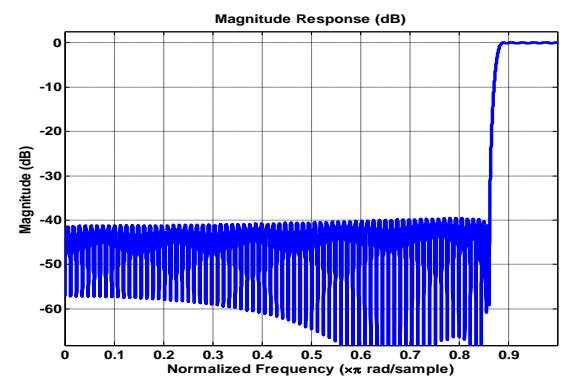

(a)MCDM-I, $M=1$

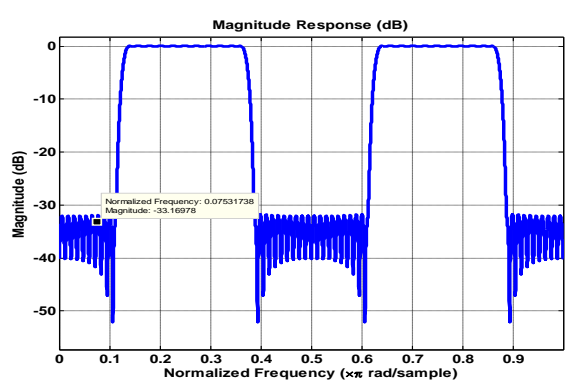

(b)MCDM-I, $M=4$

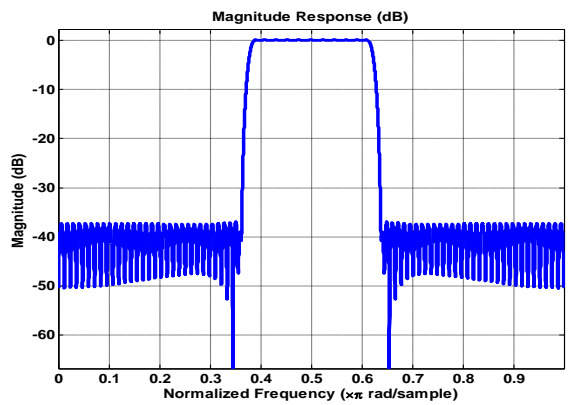

(c)MCDM-I, $M=2$

Fig 3: MCDM Operations

The ICDM-I design steps in [2] can be used to extract the five uniform channels in Fig 1using the same modal filter used in $\mathrm{CDM}$ and MCDM approaches. Giving a modal filter in Fig 2 (a), the ICDM-I operations are performed with $M=4$. The frequency response of CDM-I, $M=4$ that contains sb1, sb3 and sb5 is shown in Fig 2 (c). The frequency response for MCDM-I, $M=4$ is shown in Fig 3 (b) which contains sb2 and sb4. Two masking filters, MF1 and MF2 shown in Fig 4 (a) and 4 (b) respectively, are used in the extraction of desired subbands.

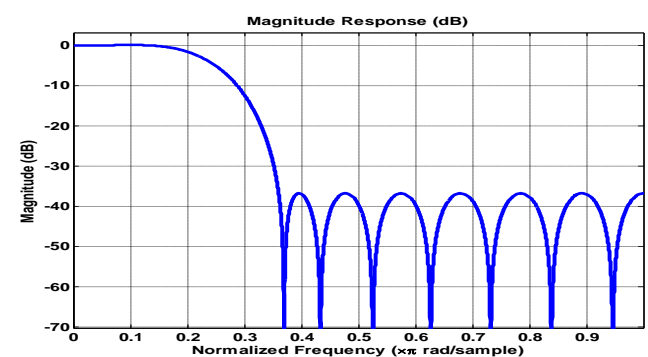

(a)MF1

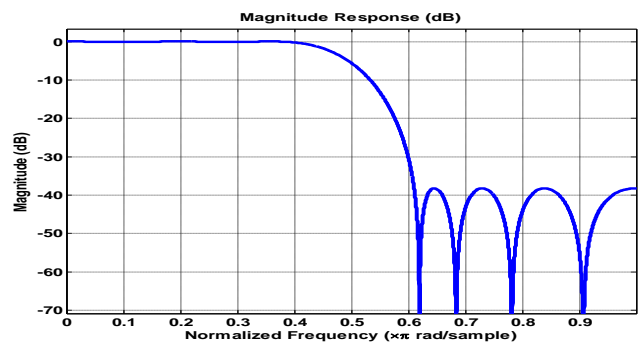

(b)MF2

Fig 4: Two Masking Filters of ICDM-I Operation

The frequency response of MF1 is used to extract sb1. Similarly, the frequency response of MF2 is used to extract sb2. Fig 5 (a) and Fig 5 (b) show the frequency responses obtained after performing MCDM-I on MF1 using $M=1$ and $M_{2}=2$ which are used to extract sb5 and sb3 responses, respectively. The frequency response in Fig 5 (c) is used to extract sb4, which represents the frequency response obtained from performing MCDM-I on MF2 using $M=1$. As a result, using a design technique of ICDM-I FB, the five channels shown in Fig 1 can be obtained.

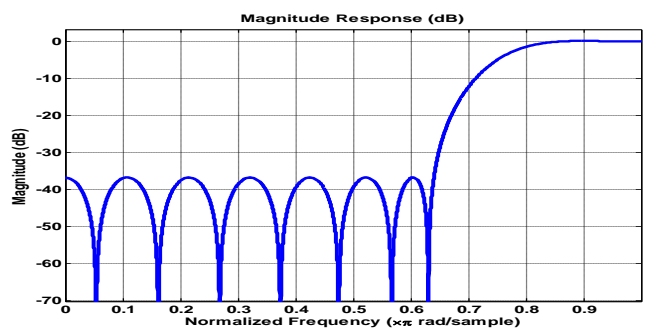

(a)MCDM-I on MF1, $M=1$

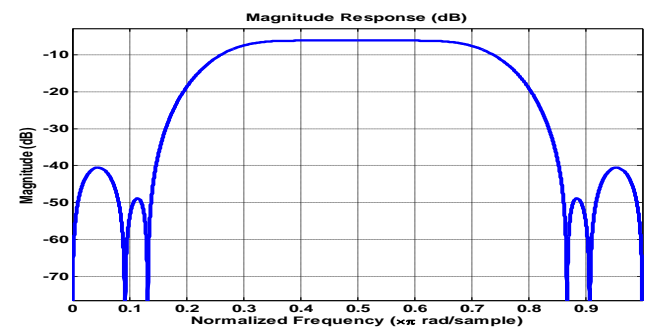

(b)MCDM-I on MF1, $M=2$

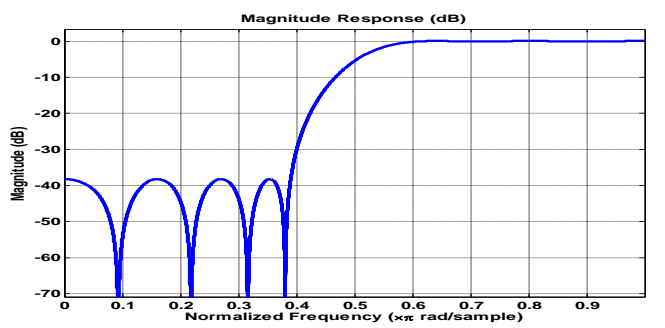

(c) MCDM-I on MF2, $M=1$

Fig 5: ICDM-I Operations on MF1 and MF2

The ICDM approach in [13] can be used to obtain the real subbands in Fig 1 using the same modal filter specifications. Perform CDM-I with $M=2$ as in Fig 2 (b). To obtain sb5, the modal filter's frequency response has to be subtracted from the frequency response of Fig 2 (b). Perform MCDM-I by $M=$ 
2 to get sb3 as shown in Fig 3 (c). Now use the complementary delays to get a complementary response of Fig 3 (c) as shown in Fig 6 (a). The frequency response of Fig 2 (b) is subtracted from the complementary response in Fig 6 (a) to obtain sb2 and sb4 together in the output as shown in Fig 6 (b), which are split by low-order masking filters of wide-TBW. Hence, the five subbands can be obtained using the ICDM-FB. The five channels are extracted using four approaches based on the coefficient decimation method and using the same modal filter specifications.

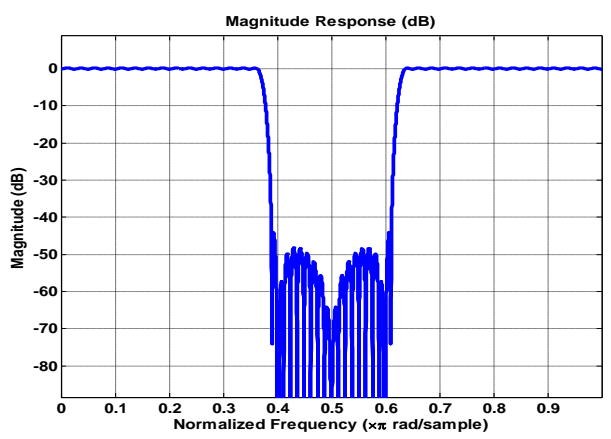

(a)Complementary response of Fig 3(c)

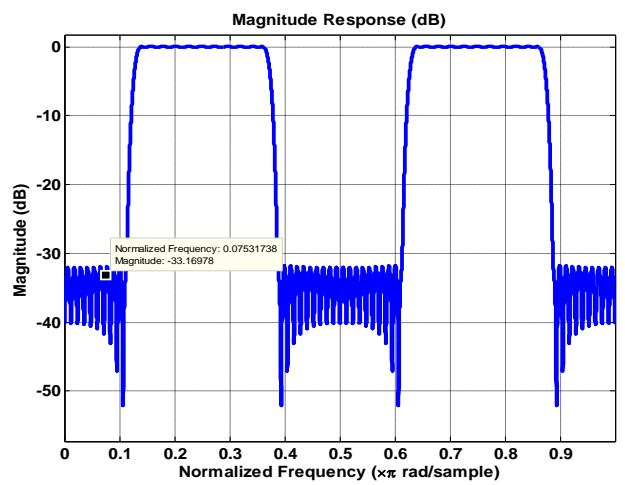

(b)Subtracting Fig 2 (b) from Fig 6 (a)

Fig 6: ICDM Operations

\subsection{Complexity Comparison}

If the DFTFB is to be used to extract the channels in Fig 1, then the complexity which depends on the involved multiplication operation number, can be represented by the sum of the length of the prototype filter $\left(l_{p}=161\right)$, and the multiplications number needed for an 8-point computation of fast Fourier transform (FFT). As direct form is followed in the DFTFB, 161 taps should be implemented. Hence, the total multiplications number of the DFTFB can be computed as $161+8 \log _{2} 8=161+24=185$. It can be noted that the complexity of this FB is increased by increased channel number.

The multiplications number required in CDFB implementation represents the sum of multiplications number required for modal filter's implementation and the multiplications number required for implementation of masking filters. Using transposed direct-form structure implementation of FIR filter, CDFB multiplication complexity is considerably lower than that of DFTFB. The modal filter of order 160 is used for obtaining sb1, whereas to obtain other channels, $M=2$ and $M=4$ are used. Hence, the maximum decimation factor involved is 4 , and the overdesigned modal filter order is 192 according to (6). The taps of the masking filters performed to get $\mathrm{sb} 4$ and $\mathrm{sb} 2$ are computed to be 19 according to (4). Hence the total multiplications number of this FB in uniform channelization is $\lceil 193 / 2\rceil+(\lceil 19 / 2\rceil * 2)=117$. However, due to the symmetry of FIR filter coefficients, only 117 taps are needed for implementation because the CDFB uses the direct form transposed FIR filters.

In case of MCDM, the same number of multiplications is required to obtain the desired subbands since the same modal filter and masking filters are used.

In ICDM-I based FB, Sb1, sb3 and sb5 can be obtained after performing CDM-I on the modal filter using $M=4$ whereas, $\mathrm{sb} 2$ and sb4 can be obtained after performing MCDM-I on the modal filter using $M=4$. All five subbands can be extracted using appropriate masking filters MF1 and MF2 of order 17, 19 respectively. The overdesigned modal filter order is 192 . The complexity of this type of FBs is represented by the number of involved multiplications. Hence the total number of multiplications for this type of $\mathrm{FB}$ is $[193 / 2]+[19 / 2]+[17 / 2]=116$ which is approximately equal to the number of multiplications involved in CDM and MCDM approaches.

In the uniform ICDM-FB, in order to obtain sb1 the modal filter itself is used. Whereas, to obtain sb5 the CDM-I is used with $M=2$. Sb3 is extracted using MCDM-I by $M=2$. Sb2 and $\mathrm{sb} 4$ are obtained after subtraction the frequency response achieved using CDM-I, with $M=2$ from the complementary response to the former response. Hence, the maximum decimation factor is 2 , and the overdesigned modal filter order is 176 according to (6). The masking filter lengths for obtaining sb2 and $\mathrm{sb} 4$ are of order 18 using (4). Hence the total multiplications number for the uniform ICDM-FB is $\lceil 177 / 2\rceil+([19 / 2\rceil * 2)=109$. Hence, the total number of multiplications involved in ICDM based FB is less than those in CDM, MCDM and ICDM-I because the maximum decimation factor involved in ICDM-FB is 2 whereas, it is 4 in the former three types of FBs. The complexity of the CDM based FBs designed to serve uniform channelization, is summarized in Table 1 .

\subsection{Flexibility Comparison}

The flexibility of a filter bank can be defined by the number and locations of distinct channels obtained from that FB. The channels of DFTFB are located at $(2 \pi / M)$ integer multiples. In the DFTFB, $M$ is fixed and its value in this example is 8 . Hence, the locations of resulting subbands are at integer multiples of $(2 \pi / 8)$. The drawback of fixed channel stacking in DFTFB makes it not suitable applicant for transceivers of cognitive radio.

In CDFB, the channels locations are located at $(2 \pi / M)$ integer multiples, and the center frequencies can be changed since $M$ is a variable. Hence, center frequencies will be shifted to another $2 \pi / M$ locations by using different $M$ values, without changing pass bandwidth.

The resolution by means of center frequency locations is $(\pi / M)$ in MCDM, which is twice the resolution obtained in CDM $(2 \pi / M)$. Thus, using MCDM, a higher degree of flexibility and reconfigurability can be achieved. The same resolution of $(\pi / M)$ can be achieved in both ICDM-I and ICDM approaches. 
Table 1. Comparison of Multiplication Complexity Design Example of Uniform channelization

\begin{tabular}{|l|l|l|l|l|l|}
\hline & DFT & $\begin{array}{l}\text { CDF } \\
\text { B }\end{array}$ & $\begin{array}{l}\text { ICD } \\
\text { M-I }\end{array}$ & $\begin{array}{l}\text { MC } \\
\text { DM }\end{array}$ & $\begin{array}{l}\text { ICD } \\
\text { M }\end{array}$ \\
\hline $\begin{array}{l}\text { Prototype/moda } \\
\text { ( filter length } \\
\left(l_{p} / l_{\text {Mod }}\right)\end{array}$ & 161 & 193 & 193 & 193 & 177 \\
\hline $\begin{array}{l}\text { Masking filter } \\
\text { length }\left(l_{\text {Mas }}\right)\end{array}$ & - & 38 & 36 & 38 & 38 \\
\hline $\begin{array}{l}\text { No. of } \\
\text { multiplications }\end{array}$ & 161 & 117 & 116 & 117 & 109 \\
\hline $\begin{array}{l}\text { No. of } \\
\text { multiplications } \\
\text { (DFT, S=8) }\end{array}$ & 24 & - & - & - & - \\
\hline $\begin{array}{l}\text { Total no. of } \\
\text { multiplications }\end{array}$ & 185 & 117 & 116 & 117 & 109 \\
\hline
\end{tabular}

\section{CONCLUSION}

Conventional spectrum sensing methods use filter bank that employs DFTFB while, in this thesis several methods based on the coefficient decimation method are designed to serve a uniform channelization for wideband spectrum sensing in cognitive radios. Four types of CDM based FB are explained in this paper with the aid of a design example. Complexity analysis regarding the design example clearly specify that the ICDM approach offers a better filter length saving compared to that of the other methods due to reduced decimation factors involved. The flexibility by means of the number of resulting subbands and their locations is better in MCDM, ICDM-I and ICDM-FBs than that of the CDM-FB.

\section{REFERENCES}

[1] P. P. Vaidyanathan, "Multirate digital filters, filter banks, polyphase networks, and applications: a tutorial". Proceedings of the IEEE, Volume 78, No. 1, pp. 56-93, August 2002.

[2] A. Ambede, K. G. Smitha, et al., "A New Low Complexity Uniform Filter Bank Based on the Improved Coefficient Decimation Method", Circuits, Systems, and Signal Processing, Volume 32, No. 6, pp. 2543-2557, December 2013.

[3] J. Mitola and G. Q. Maguire, "Cognitive radio: Making software radios more personal", IEEE Personal Communications, Volume 6, No.4 pp. 13-18, August 2002.
[4] S. Haykin, "Cognitive radio: Brain-empowered wireless communications", IEEE J. Sel. Areas Commun., Volume 23, pp. 201-220, 2005

[5] R. Mahesh and A. P. Vinod, "Coefficient decimation approach for realizing reconfigurable finite impulse response filters", Circuits and Systems

[6] M. Lin, A. P. Vinod, et al., " Very Low Complexity Variable Resolution Filter Banks for Spectrum Sensing in Cognitive Radios Using Multi-Stage Coefficient Decimation", Wireless Communications, Networking and Mobile Computing, 5th International Conference on, pp. 1-4, 2009.

[7] R. Mahesh and A. P. Vinod" Reconfigurable Discrete Fourier Transform Filter Banks for Multi-Standard Channelizers" Signal Processing and Communications (SPCOM), pp. 1-5, July 2010.

[8] A. Ambede, K. G. Smitha, et al., "A modified coefficient decimation method to realize low complexity FIR filters with enhanced frequency response flexibility and passband resolution", Telecommunications and Signal Processing (TSP), pp. 658 - 661, July 2012.

[9] A. Ambede, K. G. Smitha, et al., "An Improved Coefficient Decimation based Reconfigurable Low Complexity FIR Channel Filter for Cognitive radios" Communications and Information Technologies (ISCIT), pp. $22-27$, October 2012.

[10] R. Mahesh and A. P. Vinod, "A low-complexity flexible spectrum-sensing scheme for mobile cognitive radio terminals," IEEE Transactions on Circuits and Systems II, Volume 58, No.6, pp. 371-375, June 2011.

[11] Y. Lim, "Frequency-response masking approach for the synthesis of sharp linear phase digital filters", IEEE Transactions on Circuits and Systems, Volume 33, No.4 pp. 357- 364, January 2003.

[12] R. Mahesh and A. P. Vinod, "Low complexity flexible filter banks for uniform and non-uniform channelisation in software radios using coefficient decimation" IET Circuits, Devices \& Systems, Volume 5, No. 3, pp. 232242, May 2011

[13] A. Ambede, A. P. Vinod, et al., " Flexible Low Complexity Uniform and Nonuniform Digital Filter Banks With High Frequency Resolution for Multistandard Radios", IEEE Transactions on Very Large Scale Integration (VLSI) Systems, Volume 23, No. 4, pp. 631 - 641, March 2015.

[14] M. Bellanger, G. Bonnerot, et al., "Digitel filtering by polyphase network: application to sample rate alteration and filter banks" IEEE Transactions on Acoustics, Speech, and Signal Processing, Volume 24, No. 2, pp 109 - 114, January 2003. 